Bürgern: Jeder ist gleichzeitig Bewacher und heimlicher Beobachter. Raúl Trejo ergänzt, dass der so genannte Monitoring-Bürger

alle möglichen Themen diskutiert und mit verschiedensten Menschen in Kontakt steht. Denn er begegnet ihnen nicht nur im Fernsehen oder hört ihnen im Radio zu, sondern ist oft Teil der gleichen Netzwerke wie sie. Wenn unter den Personen, denen wir auf Twitter folgen neben dem Abgeordneten für unsere Region, dem Vorsitzenden unserer bevorzugten Partei und dem Präsidenten auch unsere ArbeitskollegInnen oder Schulfreunde, Nachbarn und alte Bekannte sind, hat sich etwas Grundlegendes in unserem Verhältnis zu öffentlichen Angelegenheiten geändert. Wir beobachten all unsere Mitmenschen sowohl in den Medien, als auch in den soziodigitalen Netzwerken. (Trejo 2015, 21)

In dieser neuen Kommunikationssituation ist der so genannte CyberUtopismus entstanden. Jeder Bürger hätte Zugang $\mathrm{zu}$ Informationen darüber, wie Politiker öffentliche Güter verwalten und verwenden und vielleicht in Korruption verwickelt sind, etwa durch Chats und Interaktionen mit anderen Bürgern auf Facebook und Twitter. Wir könnten gar das Entstehen politischer Alternativen begleiten. Kurz gesagt, würden wir uns als Bürger in unserem Zugang zu Informationen emanzipieren. Was hat nun - trotz Blogs, sozialen Netzwerken und Wikis - innerhalb und außerhalb der Logik des Internets dazu beigetragen, dass diese Illusionen heute nicht mehr gültig sind?

\title{
Was haben wir gemeinsam?
}

Diese Interaktionen zwischen Akteuren mit noch unklaren Potentialen und diffusen Eigenschaften, sowohl was Unternehmen als auch Bürger betrifft, neigen dazu, imaginative Ausdrucksformen hervorzubringen. Sie nehmen geradezu wahnhafte Formen an, wenn das Durcheinander zwischen der Expansion von Unternehmen, dem zögerlichen Handeln des Staates und den Gegenaktionen der Bürger durch das Treiben so genannter Internet-Trolls zunehmend undurchsichtiger wird: Trolls sind bezahlte Internetnutzer, die Handlungen simulieren oder andere Nutzer emotional provozieren. In einigen Fällen handelt es sich um Bots, die aus versteckten Servern fremdgesteuert werden. Sie werden von dort aus verwaltet, und die vordigitalen Regeln 
der Partizipation somit gestört. Das ist für echte Nutzer nicht selten entmutigend.

Eine Neuvorstellung des Schlüsselbegriffs Öffentlichkeit im Sinne des liberalen Gedankens ist ohne eine tiefe Reflexion der Eigenschaften, die der Liberalismus ihr einst zugeschrieben hat, unmöglich. Dazu ist eine Entschlüsselung der Kernelemente nötig, die die Beschreibungen und die Verteidigung des öffentlichen Lebens entweder überzeugend oder für eine Epoche unpassend machen. Hierzu dienen unter anderem die Ideen von Jürgen Habermas und Hannah Arendt, Ulrich Beck oder Richard Sennett. Sie geben treffende Hinweise auf die Schwächung des Staats gegenüber dem ökonomischen Markt sowie die Verlagerung des öffentlichen Bereichs in die Sphäre des Privaten. Darüber hinaus markieren sie den Anstieg deregulierter Medienunternehmen, das Pochen auf Gewinn und den klientelhaften Umgang mit den Zuschauern. Diese Entwicklungen bleiben bestehen und werden für Unmut sorgen. Es ergibt sich allerdings ein anderer Sinn dieser Entwicklungen, wenn wir uns nicht auf die politische Soziologie, auf Institutionen oder auf die industrielle Dynamik der Medienbranche, sondern auf die Erfahrung der Bürger konzentrieren. Was bedeutet der Begriff des Öffentlichen für sie? Was bedeutet »das Gemeinschaftliche« aus der Perspektive der Bürger? Immerhin lassen diese Vorstellungen uns immer noch mit einem gewissen Gefühl der Koexistenz leben, und sie führen zur Interaktion, zum Wettbewerb und manchmal noch zu Solidarität. Ohne die vielen Arten und Weisen des SichNeuerfindens als Bürger zu vernachlässigen, soll jedoch zunächst auf zwei typische Merkmale der heutigen Zeit eingegangen werden: die Prekarität und die Unsicherheit.

Eine erste Definition des Begriffs des Öffentlichen geht von einem gedanklich abstrakten Ort aus, an dem wir das Risiko eines Lebens ohne Grenzen und Regeln vermeiden oder kontrollieren möchten. In der Moderne sorgen wir uns um die Öffentlichkeit, da wir einen Ort außerhalb des Göttlichen kreieren müssen, dessen Regeln des Zusammenlebens sich nicht hinreichend aus dem Fortbestand der Familie und des Nationalstaats ergründen. Seitdem wir in einer Welt mit globalen Interdependenzen leben, die sich nicht in äquivalenten Formen und Strukturen einer globalen Regierung spiegeln, wird die Herausforderung für eine Definition dessen, was wir Menschen noch gemeinsam haben oder in welchen Punkten wir trotz einiger Unterschiede noch übereinstimmen, eine zunehmend dringendere und schwierigere Frage. Es besteht das Risiko, die Grenzen für das, was erlaubt ist, und das was nicht erlaubt ist, stetig niedriger zu setzen, indem wir immer weniger sozio- 
kulturelle und politische Kriterien festlegen, die ein Zusammenleben trotz Unterschieden ermöglichen.

Die Medien und Netzwerke erfassen die Unzufriedenheit der Bewohner des urbanen Raums. Diese finden sich nicht damit ab, inmitten von diffusen und unüberschaubaren Prozessen zu leben. Folglich kreieren das Radio, das Fernsehen und vor allem das Internet translokale Netzwerke - ortsbezogene Geschichten. Während die territoriale Expansion der Megastädte die reale Verbindung zwischen den Gruppierungen ihrer Bewohner schwächt, liefern soziale Netzwerke Informationen und Unterhaltung nach Hause: Die unkontrollierte Informationsexplosion in Richtung der Randgebiete lässt die Bewohner das Gefühl für die Grenzen »ihres« Territoriums verlieren. Ihre Abgeschiedenheit wird durch Medienberichte, WhatsApp-Nachrichten und die Übertragung der Geschehnisse an weit entfernten Orten über virtuelle Server kompensiert.

Radio und Fernsehen, die sich dazu verpflichtet haben, der Stadt Gehör zu verschaffen und ihr Kohärenz zu verleihen, gestalten ihre Kommunikationsstrategien derweilen so um, dass sie an Orten mit Wiedererkennungswert greifen: Reporte beginnen mit »Wir befinden uns hier vor dem Gebäude...«. Auch beginnen Mitteilungsfelder auf dem Handy in der Regel mit der Frage »Wo bist du gerade?«. Sogar transnationale Unternehmen wissen, dass ihre Zielgruppen von ihnen erwarten, dass die Bedeutung eines bestimmten Ortes und der dortigen Gemeinschaft zur Sprache kommt. Folglich nehmen die Medien eine Doppelrolle ein: Zum einen als makrosoziale Informanten, die die Geschehnisse an abgelegenen Orten im eigenen Land und auf der Welt weitergeben. Zum anderen als eine Art mikrosoziale Vertrauensperson, die die emotionalen Engpässe und Erschütterungen in der Stadt, sowie gleichermaßen globale Unruhen und Katastrophen wiedergeben. Demgemäß erscheinen in einer Berichterstattung die Sitten der internationalen Diplomatie, und in der anderen das private Auftreten unserer Nachbarn.

Von Interesse sind auch die jüngsten Veränderungen bei der medialen Verwaltung der öffentlichen Angelegenheiten: Zu Beginn der Radioübertragung und des Fernsehens richteten einige Sender in nationalstaatlichem Besitz ihre Inhalte im sozialen Sinn aus. Die Konzeption des öffentlichen Raums war laut John Keane an ein »Broadcast-Modell des öffentlichen Dienstes« gebunden. Keane hat aufgezeigt, wie wichtig dieses Modell in Großbritannien, den Niederlanden, in Deutschland und Kanada war. Es diente der Verhinderung von finanziellem Druck und der Einschränkung von Werbung in ihrem Umfang und ihrer Ausrichtung. Weiterhin gewährte es den Bürgern Zugriff 
auf Informationen, um sich an den jeweiligen gesellschaftlichen Debatten zu beteiligen. Mehrere Autoren haben diese befürwortende Sicht auf die medialen Errungenschaften auf den Rundfunk und das Fernsehen in Lateinamerika ausgeweitet. Dort wurden über diese Medien erste virtuelle Gemeinschaften geschaffen. Diese wurden unter anderem auch für die Herstellung eines nationalen Sinns zwischen Regierungen ohne Massenbasis und der Bevölkerung genutzt (Martín Barbero 1987; Ortiz 1988).

Als das Internet und die neuen Medien begannen, ihre Wirkung zu entfalten, wurden sie als die neuen Agorai bezeichnet, als Orte der Masseninformation. Diese Beschreibung trifft teilweise zu. Durch die Kommunikationsmedien, wie heute in den sozialen Netzwerken, empfangen wir einen Großteil der Berichterstattungen und Nachrichten, beobachten unterschiedliche Meinungsäußerungen über sie und beteiligen uns selbst an den Diskursen. Während die politischen Parteien ihre Glaubwürdigkeit und die Fähigkeit zur Vertretung öffentlicher Interessen immer mehr verlieren, beginnen die Medien mit der Neubesetzung dieser Vermittlerrolle und steigen in die soziale Debatte ein.

Dies legt die Frage nahe, wie sich diese ehemals als Agorai bezeichneten Räume angesichts der Multiplikation an Informationen durch die Kommunikation auf städtischer, nationaler und transnationaler Ebene transformieren. Es entsteht ein Gefühl der Hyperinformation. Gleichzeitig beeinflussen uns so viele Prozesse, die uns zwar betreffen, aber die wir nicht kontrollieren können. Dies liegt etwa an ihrem Ausmaß, ihrer schnellen Überholung oder an der Tatsache, dass ihre Logik an abgelegenen, für uns nicht einsehbaren Schauplätzen entschieden oder verwaltet wird.

So verändert sich die Erfahrung rund um das, was wir konstruieren und entscheiden können. Das Gefühl der Dekonstruktion und der Unfähigkeit zum Entscheiden (d.h. die Unregierbarkeit) suggeriert, dass nur noch Fragmente von dem, was uns Menschen noch verbindet, entscheidend für die Konfiguration der heutigen Welt sind. Diese Fragmente werden zudem bloß noch verwaltet und die Orte, an denen Entscheidungen getroffen werden, sind für uns nicht unzugänglich. Da wir uns weiterhin nach verständlichen Zusammenhängen und klaren Rahmenbedingungen sehnen, an denen wir festhalten können, imaginieren wir einerseits Minderheiten, Ausländer und abstrakte Begriffe wie der Imperialismus einerseits als die Schuldigen für die Unordnung. Anderseits sehnen wir uns nach rettenden Instanzen der Anerkennung und Solidarität, wie etwa den sozialen Netzwerken. Je weiter entfernt und je undurchsichtiger das Wissen über die Mächtigen der Welt scheint, des- 
to bunter werden die Vorstellungen über sie. Nur vereinzelt gelingt es Menschen, alternative Formen der Resignation und der Kritik zu äußern, indem sie die Machtverhältnisse der großen nationalen und transnationalen Akteure grundsätzlich in Frage stellen.

Vor etwa zwanzig Jahren wurden Studien durchgeführt, die zivilgesellschaftliche Organisationsformen und deren Potenzial für einen gesellschaftlichen Wandel im Übergang zu einem neuen Zeitalter evaluieren sollten. Dabei wurde vor allem die Ungleichheit im Zugang zu Informationen und Ressourcen bei lokalen Akteuren festgestellt. So sehr NGOs den Versuch unternommen haben, über Plattformen oder transnationale Netzwerke einzugreifen und sich mit der Diversität vor Ort zu verbinden, so sehr mussten sie erkennen, dass die Bedürfnisse und die Handlungsstile der einzelnen Gesellschaften eher entlang globaler thematischer Moden interpretiert wurden als auf der Grundlage einer gemeinsamen Diagnose der realen Umstände. Die bürokratische Logik der multilateralen Kooperation und der Banken, sowie die Abhängigkeiten verschiedener Organismen vom System der Vereinten Nationen, sind längst überall angekommen. Diese sind eben keine de-territorialen, sondern transterritoriale Organismen, die für die Produktion von Diskursen verantwortlich sind und in Bezug auf spezifische soziale Kontexte handeln (Abéles 2008; Mato 2004). Ihre Vorherrschaft zeigt sich auch im globalen System der audiovisuellen Produktion (Kino oder Fernsehen) und ist im Internet noch stärker ausgeprägt. In der elektronischen Agora wird versucht, "von unten« zu handeln und durch mehrdimensionale Bürgernetze sinnvolle Verbindungen zu schaffen (Winocur 2002). Diese Versuche brachten es bisher zu dem ersten Schritt, Gemeinschaften zu vernetzen und den digitalen Umweltaktivismus voranzutreiben, sowie für mehr Horizontalität im Internet im Sinne einer emanzipatorischen Verbindung zu sorgen. Ein Beispiel für diese Expansion lokaler Auseinandersetzungen ist der Zapatismo.

Für individuelle und institutionelle Akteure hat sich die Situation lokal, national und transnational verändert, seit wir in einer algorithmischen Gouvernementalität leben. Die vorherige Phase der statistischen Regierungsführung sortierte die Daten der Menschen, die an Befragungen teilgenommen und damit die von der Regierung, einer Partei, einer Firma oder einer sozialen Organisation zu bestimmten Zwecken erbetene Information preisgegeben hatten. Ausgehend von dieser Sichtweise sammeln Regierungen Daten, um

für Sicherheit, Kontrolle, Ressourcenmanagement und optimierte Ausgaben zu sorgen [...]; private Unternehmen erheben Daten für Marketing- 
und Werbezwecke, individualisierte Angebote, die letztendlich auch die Lagerverwaltung und das gesamte Dienstleistungsangebot verbessern. Dies geschieht hinter der Idee, ihre kommerzielle Effizienz und folglich ihre Einnahmen zu erhöhen...; Wissenschaftler sammeln Daten zur Gewinnung und Verbesserung von Wissen. (Rouvroy und Berns 2016, 92)

Im Gegensatz dazu werden im Rahmen der algorithmischen Expansion Millionen verstreuter Daten, die bis zu einem gewissen Punkt unabhängig von den Anwendungen, den Nutzern oder kollektiven Organismen vorliegen, korreliert. Dabei nutzten diese Anwendungen und Organismen die Daten ursprünglich bloß für soziodigitale Zwecke. Die automatisierte Produktion von Wissen erfordert fast kein menschliches Eingreifen mehr, und »kann daher auf jede Form früherer Hypothesen (im Gegensatz zu traditionellen Statistiken und ihrer klassischen Verifikation einer Hypothese) verzichten, also jede Form der Subjektivität vermeiden« (Ibid., 93).

Diese Unterscheidung zwischen Regierungsmodi und verschiedenen Kommunikationsstadien verdichtet die im ersten Kapitel angedeutete Kritik am Begriff des Populismus. Die Bandbreite verschiedener Prozesse schlicht und einfach als Populismus $\mathrm{zu}$ bezeichnen, bedeutet, die Geschichte als Wiederholung der Beziehungsformen zwischen der Elite und der Masse zu denken. Schon vor 70 Jahren wurde dies übersehen, als versucht wurde, Mussolini und Perón zu vergleichen. Und dies gilt auch heute für die Gleichsetzung von Führungsfiguren, die zwar nicht in länger vergangenen doch durchaus unterschiedlichen historischen Kontexten aktiv waren, wie etwa Berlusconi, Chávez und Trump.

Unter anderem führen zwei Fehler zu diesen schwer haltbaren Assoziationen: Erstens wird nicht beachtet, dass Führungsfiguren größere Foucaultianer sind, als ihre Analysten, und - kurz gesagt - ihre Macht nicht von oben nach unten ausüben: Ihr politisches Geschick leitet sich vielmehr aus dem Verständnis ab, dass es bei Macht um das Erkennen einer strategischen Chance geht, durch die man politische Kräfte aufteilt und sie zum richtigen Zeitpunkt wirken lässt. Zweitens ist die Autonomisierung des politischen Konflikts, wie weiterhin an Begriffen oder Aufteilungen in Pro- oder AntiCastro, in Peronismus oder Anti-Peronismus zu erkennen ist, eine träge Verlängerung der wirtschaftlichen und politisch-kulturellen Konfiguration, die dem vergangenen Jahrhundert angehört. Die strategische Lage der Politik hat sich mit der Existenz des Internets so sehr verändert wie der Rest der Gesellschaft(en). Mit dem Internet als alltäglichem Gegenstand, dem Zugang für al- 
le ins World Wide Web und dem Vormarsch der sozialen Netzwerke und dem Datenmarkt hat sich unsere Welt grundlegend gewandelt. Wir blicken auf einen Wandel ausgehend von einer Ära, in der die Gouvernementalität klassisch statistisch aufgebaut war, hin zu einer Zeit, in der der Konsens durch Algorithmen hergestellt wird.

\section{Regieren oder den Schaden in Grenzen halten?}

In diesem Kapitel soll der Frage nachgegangen werden, in welche Arten von soziokultureller und politischer Überlastung die Anhäufung von Information und Nachrichten, der Überfluss an Kommunikation und deren andauernde inhaltliche und technologische Überholung münden. Angesichts dieser Prozesse bekräftigt sich die Vorstellung, dass es anstelle von Prozessen heute häufiger um isolierte Ereignisse geht; statt von Geschichte ist die Rede von Bewegungen oder der Turbulenzen auf dem Kreditmarkt. Anstelle von gut formulierten Nachrichtenmeldungen lesen wir von »Neuigkeiten aus Politik, Wirtschaft und Werbung«. Unsere Computer und Smartphones laufen mit mehr und mehr Apps, die in einem Augenblick entsorgt und gegen neue ausgetauscht werden können.

Was geschieht mit Akteuren wie Unternehmen, beziehungsweise den nicht staatsbürgerlichen, nicht nationalen oder nicht globalisierten? Sie tragen ihre privaten Absichten in getarnter Form als öffentlich oder neutral nach außen und halten sich an gemeinsame Absprachen. Diese Rituale haben eher den Zweck, Gewinne und Umsatz zu erzeugen, als dass sie der Aufrechterhaltung des Betriebs dienen. Es gibt nur eine Handvoll großer Akteure mit wenig sichtbaren langfristigen Strategien. Sie messen unsere Meinungen und Vorlieben mit dem angeblichen Zweck, »die Kundenwünsche« (und fast nie die Bedürfnisse) zu kennen, und im Falle der Unzufriedenheit oder bei Mängeln so genannte »Schadensbegrenzung« zu gewährleisten. Ein Großteil der Arbeit solcher Meinungserhebungen bezieht sich auf diese beiden Aktivitäten. Die Wirksamkeit dieser beiden Erkennungsmethoden ist zweifelhaft, wenn wir sie auf soziale Missstände oder auf den Zerfall der Politik beziehen. Sie zeichnen sich durch eine Unbeständigkeit der Wähler- und Konsumvorlieben, die Krise des Fernsehens und der so genannten digitalen Server, der Unterminierung von Parteiführern, sowie durch instabile Pakte zwischen Regierungen, Unternehmern und uns Bürger-Verbrauchern aus. 